\title{
A NOVEL THREE-LEGGED 6-DOF PARALLEL ROBOT WITH SIMPLE KINEMATICS
}

\author{
Edelvays Cherchelanov, Ilian A. Bonev \\ École de technologie supérieure, Montreal, QC, Canada \\ E-mail: ilian.bonev@etsmtl.ca
}

\begin{abstract}
This paper presents a novel three-legged 6-DOF (degrees of freedom) parallel robot with simple kinematics. The main idea behind this novel architecture is that each of the three identical legs is controlled by two prismatic actuators with parallel directions. As a result, it is possible to control simultaneously or separately the position and the orientation of a leg. The reduced number of legs leads to a simple mechanical design with reduced risk for mechanical interferences.
\end{abstract}

Keywords: Cartesian parallel robot, direct kinematics, workspace, PKM.

\section{UN NOUVEAU ROBOT PARALLELE À SIX DDL, AVEC TROIS JAMBES ET UN MODÈLE CINÉMATIQUE SIMPLE}

\section{RÉSUMÉ}

Cet article présente un nouveau robot parallèle à trois à six ddl (degrés de liberté), avec trois jambes et un modèle cinématique simple. L'idée principale de cette nouvelle architecture est que chacune des trois jambes identiques est contrôlée par deux actionneurs linéaires dont les directions sont parallèles. De ce fait, il est possible de contrôler simultanément ou séparément la position et l'orientation d'une jambe. Le nombre réduit de jambes permet une conception mécanique simple, avec un faible risque d'interférences mécaniques.

Mots-clés : Robot parallèle cartésien, cinématique directe, espace de travail, PKM. 


\section{INTRODUCTION}

Many robots with parallel kinematics have been invented but none of them is as simple as the Cartesian parallel robot [1], invented in 2001, also known as Tripteron [2]. The kinematic model of the latter is simply

$$
x=\rho_{1}, y=\rho_{2}, z=\rho_{3},
$$

where $\rho_{1}, \rho_{2}$, and $\rho_{3}$ are the active-joint variables, and $x, y$, and $z$ are the position coordinates of the mobile platform.

Tripteron was a starting point for developing a whole new family of parallel robots. The concept was first extended to a four-legged robot with four DOFs (degrees of freedom), called Quadrupteron [3], but its direct kinematic problem requires the solution of a quadratic equation. Then, the next member of the Cartesian parallel robot family was invented, the Pentapteron. The latter [4,5] has 5 DOFs but its direct kinematic problem allows at least 208 real solutions [6]. Later, a 6-DOF member was presented [7], the Hexapteron, which surprisingly has simple direct and inverse kinematics.

In this article, we propose a new 6-DOF Cartesian robot with only three legs (Fig. 1). Whereas the design of Hexapteron's legs is intricate, requiring complex 5-axis machining, in order to avoid mechanical collisions, our novel architecture allows the design to be kept simple and requires only 3-axis machining.

First, we study the kinematic equations of the new robot, which are still trivial as in the case of the Hexapteron. Second, we analyze the workspace of the robot. Finally, we show how to transform our architecture easily to 5-DOF and 4-DOF alternatives or other 6-DOF designs while keeping the simplicity of the kinematic equations.

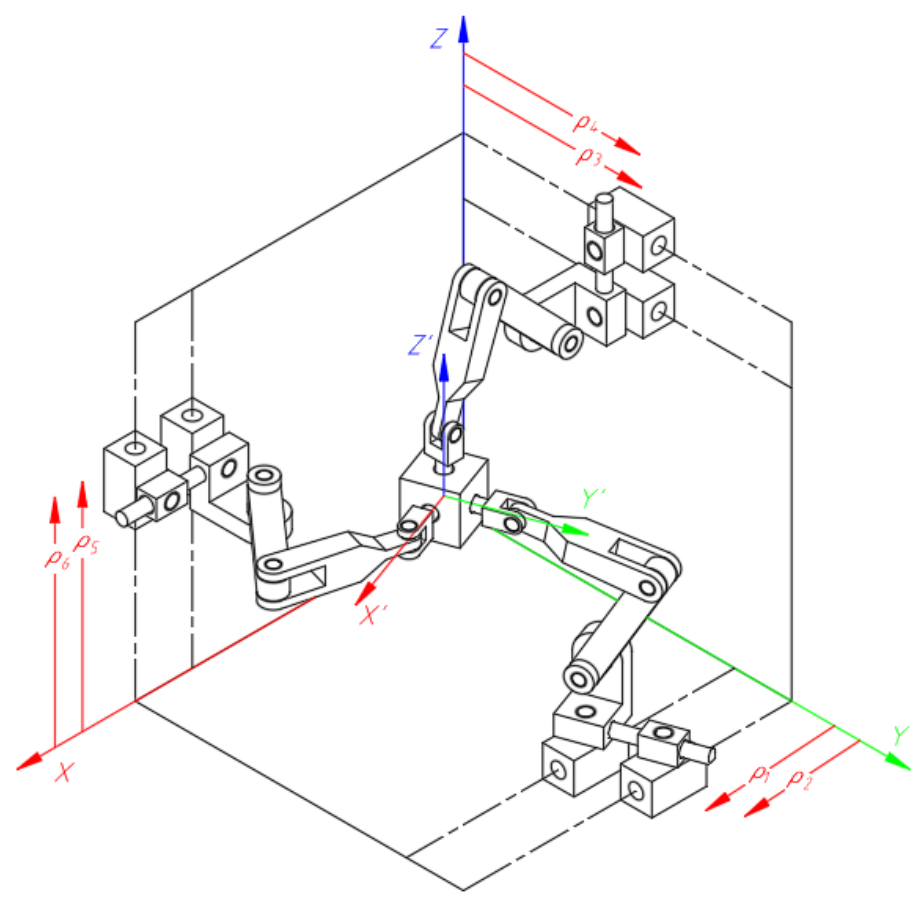

Fig. 1. Schematic of the novel 6-DOF parallel robot. 


\section{KINEMATIC MODELLING OF THE ROBOT}

The schematic of the novel architecture is shown in Fig. 1. Legs 1, 2, and 3 are each attached to the base by a set of two active prismatic joints with directions parallel to the $x, y$ and $z$ axis of the base (Cartesian) reference frame, respectively. Each of the two carriage blocks of the first, second, and third pair of linear guides are connected to secondary blocks through passive revolute joints with axes parallel to axes $z, x$, and $y$, respectively. The outer block of each pair of secondary blocks (i.e., the secondary block of legs 2, 4, and 6) is connected to the inner block through a passive prismatic joint, the direction of which is normal to the axes of the two passive revolute joints just described. This type of actuation has already been used in [7]. Then, the proximal link of each leg is connected to the inner secondary block and to the corresponding distal link via revolute joints, the axes of which are parallel to each other, and normal to the axes of the passive revolute joints previously described. Finally, legs 1, 2, and 3 are each connected to the mobile platform via a passive universal joint, the first axis of which is parallel to the axes of the preceding two revolute joints, and the second axis is along the $y, z$ and $x$ axis of the mobile (Cartesian) reference frame, respectively.

To illustrate the behaviour of a leg, we can distinguish three separate cases. The first case is when the difference of two adjacent actuator (active-joint) variables is constant. As a result, the plane of the leg (see Fig. 2) will only translate along the direction of the corresponding prismatic joint. The second case is when the difference of the two active-joint variables varies and both secondary blocks in a leg move with respect to one another. Then the plane of the leg will translate and simultaneously rotate about the axis of the passive revolute joint in the inner secondary block.

Let $\pi_{1}, \pi_{2}$, and $\pi_{3}$ be the leg planes (Fig. 2), each normal to the axes of the three intermediate revolute joints and passing through the axes of the other three revolute joints of a leg. Thus, $\pi_{1}$ contains axis $y^{\prime}$ of the mobile reference frame and is parallel to axis $z$ of the base reference frame, $\pi_{2}$ contains axis $z^{\prime}$ and is parallel to axis $x$, and $\pi_{3}$ contains axis $x^{\prime}$ and is parallel to axis $y$.

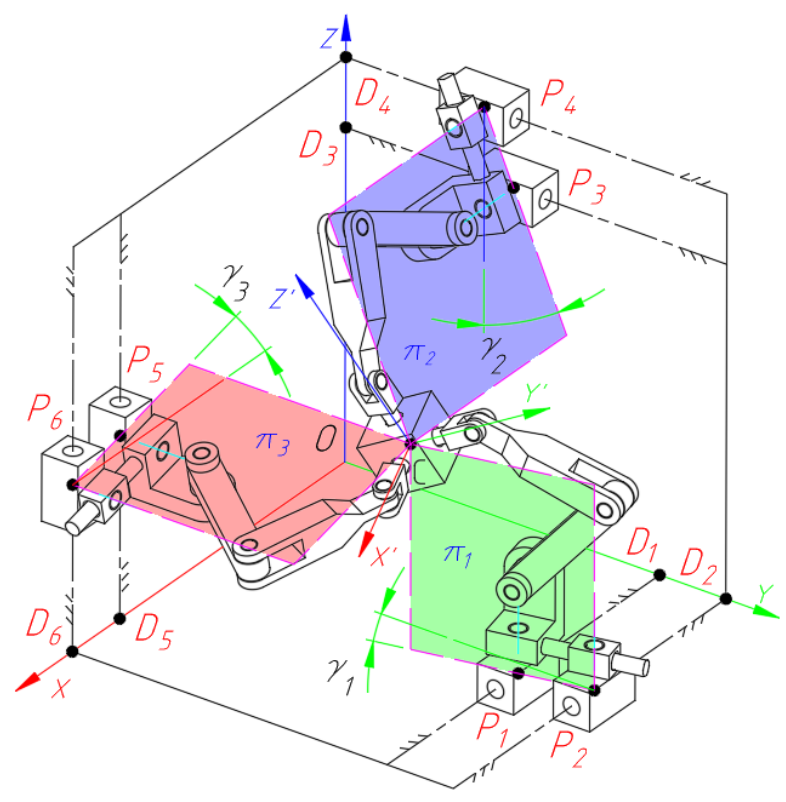

Fig. 2. Schematic of the proposed parallel robot showing the leg planes $\pi_{i}$. 

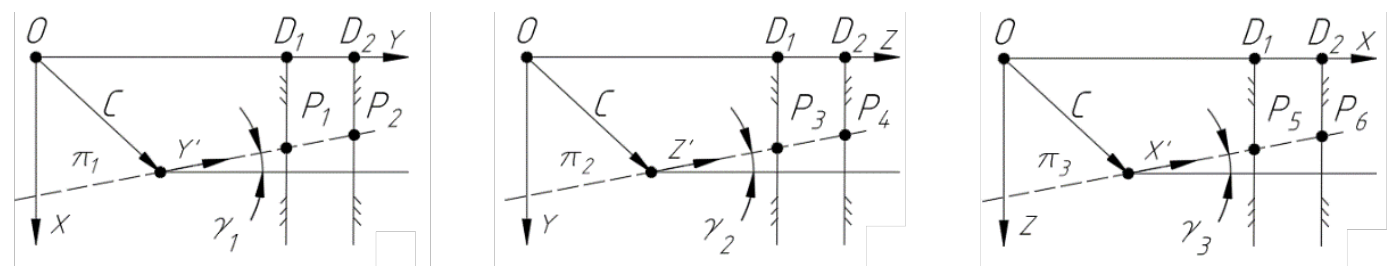

Fig. 3. Projections of the auxiliary planes.

For clarity, refer to Fig. 3, which shows the projections of the leg planes onto the base reference frame planes. For the inverse kinematic problem, we have to find the length of segments $D_{i} P_{i}$, i.e., the active-joint variables $\rho_{i}$ (in this paper $i=1,2, \ldots, 6$ ). Let $\gamma_{1}, \gamma_{2}$, and $\gamma_{3}$ be the angles between planes $\pi_{1}, \pi_{2}$ and $\pi_{3}$ and the planes $O-y z, O-x z$, and $O-x y$, respectively (see Figs. 2 and 3). Thus, we have:

$$
\begin{aligned}
& \gamma_{1}=\operatorname{atan} 2\left(r_{1,2}, r_{2,2}\right), \\
& \gamma_{2}=\operatorname{atan} 2\left(r_{2,3}, r_{3,3}\right), \\
& \gamma_{3}=\operatorname{atan} 2\left(r_{3,1}, r_{1,1}\right),
\end{aligned}
$$

where $r_{1,1}, r_{1,2}$, etc., are the elements of the rotation matrix $\mathbf{R}$ representing the orientation of the mobile reference frame with respect to the base reference frame.

Let $x, y$, and $z$ be the coordinates of point $C$ with respect to the base reference frame. Finally, let $d_{i}$ be the distance between point $D_{i}$ and the origin of the base reference frame, and let $d_{1}=d_{3}=d_{5}$ and $d_{2}=d_{4}=d_{6}$.

Thus, it can be easily seen that the active joint variables are calculated as follows:

$$
\begin{aligned}
& \rho_{1}=x+\left(d_{1}-y\right) \tan \gamma_{1}, \\
& \rho_{2}=x+\left(d_{2}-y\right) \tan \gamma_{1}, \\
& \rho_{3}=y+\left(d_{1}-z\right) \tan \gamma_{2}, \\
& \rho_{4}=y+\left(d_{2}-z\right) \tan \gamma_{2}, \\
& \rho_{5}=z+\left(d_{1}-x\right) \tan \gamma_{3}, \\
& \rho_{6}=z+\left(d_{2}-x\right) \tan \gamma_{3} .
\end{aligned}
$$

To solve the direct kinematics, we first need to find the angles $\gamma_{1}, \gamma_{2}$, and $\gamma_{3}$ from the following equations:

$$
\begin{aligned}
& \gamma_{1}=\operatorname{atan} 2\left(\rho_{2}-\rho_{1}, d_{2}-d_{1}\right), \\
& \gamma_{2}=\operatorname{atan} 2\left(\rho_{4}-\rho_{3}, d_{2}-d_{1}\right), \\
& \gamma_{3}=\operatorname{atan} 2\left(\rho_{6}-\rho_{5}, d_{2}-d_{1}\right) .
\end{aligned}
$$


Then, we can find the position of the mobile platform by solving the system of linear equations (5), (7) and (9):

$$
\begin{aligned}
& x=\frac{d_{1} t_{1} t_{2} t_{3}+\left(d_{1}-\rho_{5}\right) t_{1} t_{2}+\left(d_{1}-\rho_{3}\right) t_{1}-\rho_{1}}{t_{1} t_{2} t_{3}-1}, \\
& y=\frac{d_{1} t_{1} t_{2} t_{3}+\left(d_{1}-\rho_{1}\right) t_{2} t_{3}+\left(d_{1}-\rho_{5}\right) t_{2}-\rho_{3}}{t_{1} t_{2} t_{3}-1}, \\
& z=\frac{d_{1} t_{1} t_{2} t_{3}+\left(d_{1}-\rho_{3}\right) t_{1} t_{3}+\left(d_{1}-\rho_{1}\right) t_{3}-\rho_{5}}{t_{1} t_{2} t_{3}-1},
\end{aligned}
$$

where $t_{1}=\tan \gamma_{1}, t_{2}=\tan \gamma_{2}$ and $t_{3}=\tan \gamma_{3}$. Obviously, there is always a single solution for the position of the mobile platform, except when $\tan \gamma_{1} \tan \gamma_{2} \tan \gamma_{3}=1$. The latter corresponds to a singularity, when the three planes $\pi_{1}, \pi_{2}$ and $\pi_{3}$ intersect at a common line (note that it is impossible for any two planes to coincide). Fortunately, for a typical mechanical design, the absolute values of angles $\gamma_{1}, \gamma_{2}$, and $\gamma_{3}$ are less than $45^{\circ}$, so our mechanism is never at a singularity.

Now, it is relatively easy to see that the rotation matrix $\mathbf{R}$ has the following form:

$$
\mathbf{R}=\left[\begin{array}{ccc}
\sin \alpha_{3} \cos \gamma_{3} & \sin \alpha_{1} \sin \gamma_{1} & \cos \alpha_{2} \\
\cos \alpha_{3} & \sin \alpha_{1} \cos \gamma_{1} & \sin \alpha_{2} \sin \gamma_{2} \\
\sin \alpha_{3} \sin \gamma_{3} & \cos \alpha_{1} & \sin \alpha_{2} \cos \gamma_{2}
\end{array}\right],
$$

where $\alpha_{1}$ is the angle between the mobile $y^{\prime}$ axis and the base $z$ axis, $\alpha_{2}$ is the angle between the mobile $z^{\prime}$ axis and the base $x$ axis, and $\alpha_{3}$ is the angle between the mobile $x^{\prime}$ axis and the base $y$ axis. For a practical design, these angles are close to $90^{\circ}$ and could never be $0^{\circ}$ or $180^{\circ}$, i.e., their sines are always positive. Multiplying each two columns of the rotation matrix and dividing by the corresponding $\sin \alpha_{j}$ $(j=1,2,3)$, yields the following three equations (note that $\sin \alpha_{j} \neq 0$, since $\alpha_{j}$ can never be $0^{\circ}$ or $180^{\circ}$ ):

$$
\begin{aligned}
& \sin \gamma_{3} \cot \alpha_{1}+\cos \gamma_{1} \cot \alpha_{3}+\cos \gamma_{3} \sin \gamma_{1}=0, \\
& \sin \gamma_{2} \cot \alpha_{3}+\cos \gamma_{3} \cot \alpha_{2}+\cos \gamma_{2} \sin \gamma_{3}=0, \\
& \sin \gamma_{1} \cot \alpha_{2}+\cos \gamma_{2} \cot \alpha_{1}+\cos \gamma_{1} \sin \gamma_{2}=0 .
\end{aligned}
$$

Finally, we can solve the above three equations for the cotangents and obtain:

$$
\begin{aligned}
& \cot \alpha_{1}=\frac{\sin \gamma_{1} \cos \gamma_{1} \sin \gamma_{3} \cos \gamma_{2}-\cos \gamma_{3} \sin \gamma_{2}}{\sin \gamma_{1} \sin \gamma_{2} \sin \gamma_{3}+\cos \gamma_{1} \cos \gamma_{2} \cos \gamma_{3}}, \\
& \cot \alpha_{2}=\frac{\sin \gamma_{2} \cos \gamma_{2} \sin \gamma_{1} \cos \gamma_{3}-\cos \gamma_{1} \sin \gamma_{3}}{\sin \gamma_{1} \sin \gamma_{2} \sin \gamma_{3}+\cos \gamma_{1} \cos \gamma_{2} \cos \gamma_{3}}, \\
& \cot \alpha_{3}=\frac{\sin \gamma_{3} \cos \gamma_{3} \sin \gamma_{2} \cos \gamma_{1}-\cos \gamma_{2} \sin \gamma_{1}}{\sin \gamma_{1} \sin \gamma_{2} \sin \gamma_{3}+\cos \gamma_{1} \cos \gamma_{2} \cos \gamma_{3}} .
\end{aligned}
$$


As we already mentioned, since the absolute values of the angles $\gamma_{1}, \gamma_{2}$, and $\gamma_{3}$ are less than $45^{\circ}$, the denominator in the above expressions is always non-zero. From these three equations, we can find $\alpha_{1}$, $\alpha_{2}$ and $\alpha_{3}$, and therefore find the rotation matrix $\mathbf{R}$, which concludes the direct kinematic problem.

\section{WORKSPACE}

For the studied architecture, each leg is able to perform two types of motions, a linear motion and a rotation about an axis perpendicular to the direction of its prismatic joint. When the mobile platform has zero orientation, the robot behaves exactly as the Tripteron. The only difference is that even for pure translations along $x, y$ and $z$ axes, all six motors are controlled in pairs. In this mode, all legs move only linearly along the rails, without simultaneous rotation. Thus, for the pure translation mode, the workspace is a cube with a side equal to the stroke of the actuators, or slightly less (when the platform is rotated).

For 6-DOF applications such as pick-and-place or assembly, it is impossible to represent the orientation workspace of the robot. Referring to Eq. (17) and Fig. 2, we can obtain some rough indications about the ability to rotate about some axes, since one of the main mechanical limits is the limit on angles $\gamma_{1}, \gamma_{2}$, and $\gamma_{3}$. However, since we will be interested in orienting a specific tool reference frame, not the mobile $C-x^{\prime} y^{\prime} z^{\prime}$ reference frame, it is impossible to discuss the orientation workspace of a general case.

For machining or additive manufacturing applications, however, we are only interested in two orientation DOFs (the rotation about the axisymmetric tool is redundant). For such five-axis applications, the tool must be placed as in Fig. 4, in order to gain advantage of the inherent symmetry of the parallel robot. A video illustrating the proposed robot design is available at https://youtu.be/DXv2qoMFvEI.

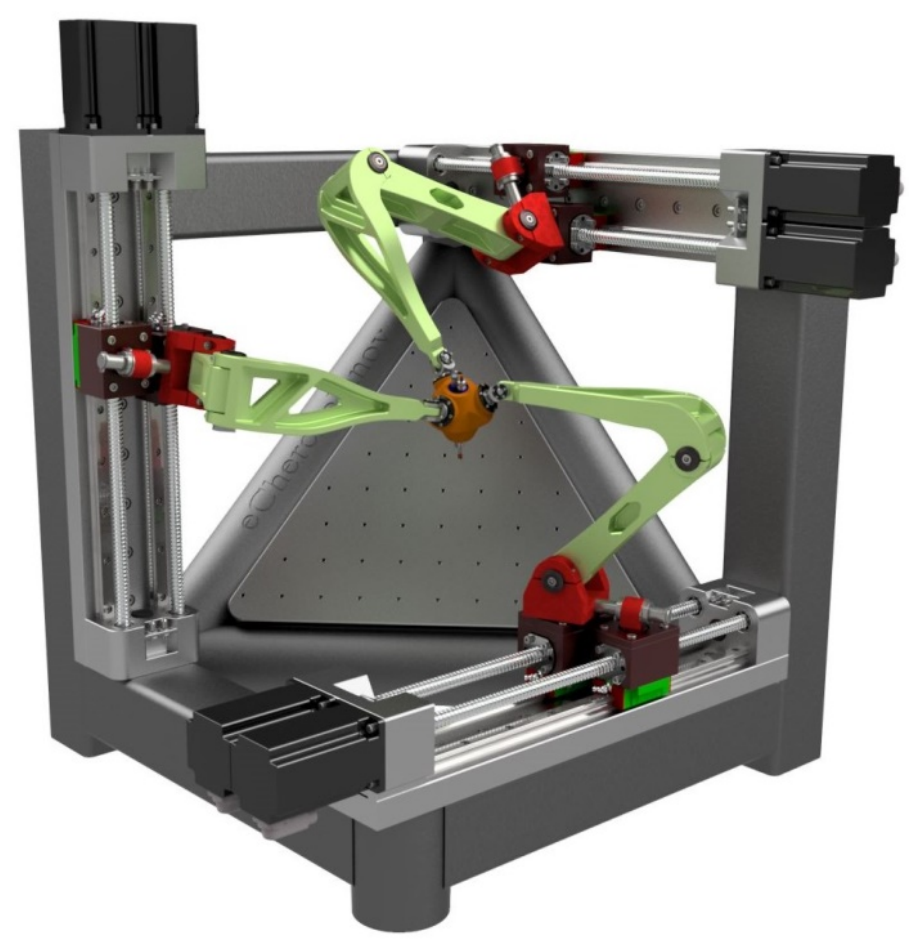

Fig. 4. Proposed mechanical design of the novel parallel robot, for machining applications. 


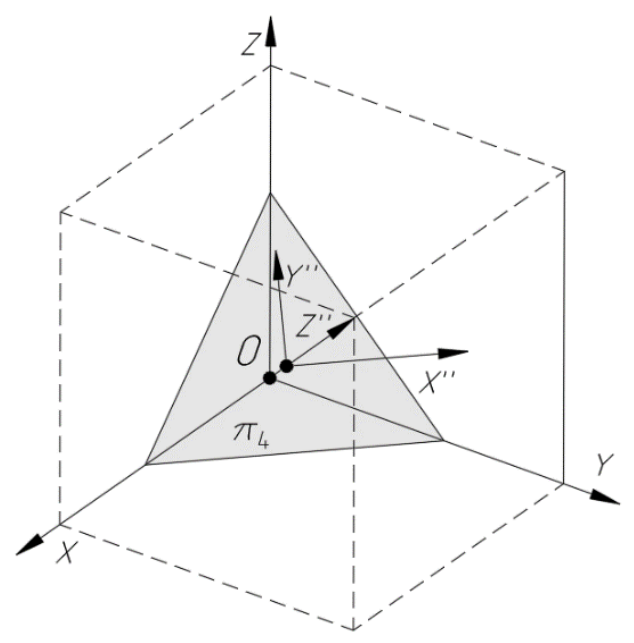

(a)

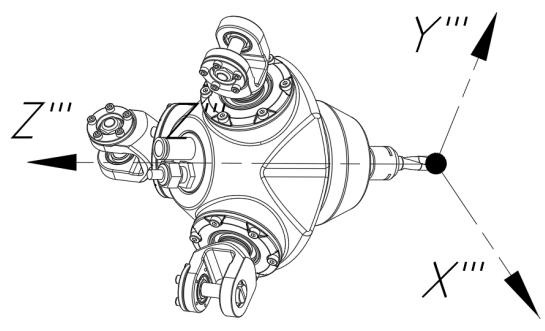

(b)

Fig. 5. New base and mobile reference frames for the robot shown in Fig. 4.

For representing the workspace of the specific implementation shown in Fig. 4 we will therefore need a new set of base and mobile reference frames. These are defined in Fig. 5. Finally, we will use the Tilt and Torsion angles [9] to represent the orientation of the mobile platform. An orientation is defined with these angles as follows: From its zero orientation, rotate the mobile reference $x^{\prime \prime \prime} y^{\prime \prime \prime} z^{\prime \prime \prime}$ about an axis in the $x^{\prime \prime \prime} y^{\prime \prime \prime}$ plane, making an angle $\phi$ with axis $x^{\prime \prime \prime}$, at $\theta$ (the tilt angle), and then rotate about the $z^{\prime \prime \prime}$ axis at $\sigma$ (the torsion angle). As shown in [9], for symmetric parallel robots such as the one shown in Fig. 4, maximum tilt is obtained at torsion angles that are very close to zero. Therefore, we can greatly simplify the workspace analysis and the operation of the robot by keeping the torsion angle equal to zero. Finally, for a given position of the tooltip, although the mobile platform can tilt in some directions more than in others (i.e., for some angles $\phi$ ), we are interested in the maximum tilt angle that can be achieved in any direction (i.e., for any angle $\phi$ ).

Unfortunately, it is impossible to devise a geometric algorithm for computing the maximum tilt angle for a given tooltip position. We therefore use a simple discretization algorithm, and for each tooltip position of a Cartesian grid, we vary $\phi$ from $0^{\circ}$ to $360^{\circ}$, and for each $\phi$, we increase $\theta$ from $0^{\circ}$ until a mechanical interference is reached. The mechanical limits that we test for are angles $\gamma_{1}, \gamma_{2}$, and $\gamma_{3}$ $\left(40^{\circ}\right)$ and the actuator strokes $(500 \mathrm{~mm})$.

Figure 6 presents the results, by displaying twelve different "horizontal" sections of the position workspace of the proposed robot. For each section, a color map is used to show the values for the maximum tilt angles.

\section{ALTERNATIVE DESIGNS}

Although the studied architecture has six DOFs, architectures with fewer DOFs might also be achieved using the same main idea. Conventional spatial PKMs (parallel kinematic machine tools) have five degrees of freedom: three translational DOFs and two rotational. The axis for the third rotational movement coincides with the rotational movement of the cutting tool. So for reducing the mechanical complexity and cost, most PKM are built without the ability for third rotational DOFs. 

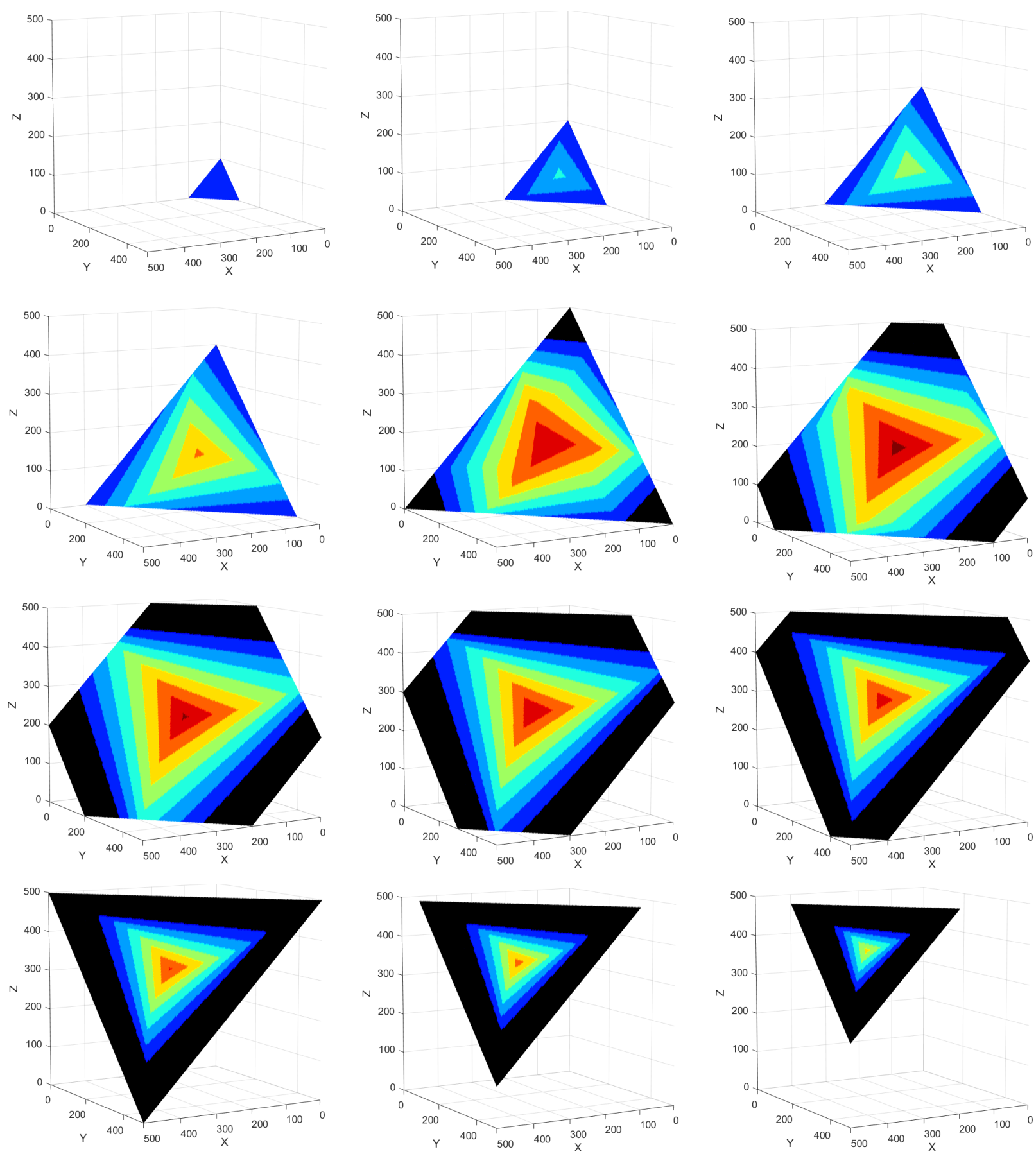

Fig. 6. Maximum tilt angle at different sections of the position workspace of the proposed robot.

In Fig. 7(a), we propose a 5-DOF version that can be used for machining or additive manufacturing. The 5-DOF version is achieved by removing motor 4 and blocking the rotation of leg 1 . Thus, leg 1 is only able to translate along its rail. Finally, we can transform our 6-DOF robot to a 4-DOF alternative, as shown in Fig. 7(b). This kind of architecture is useful for pick and place operations. 


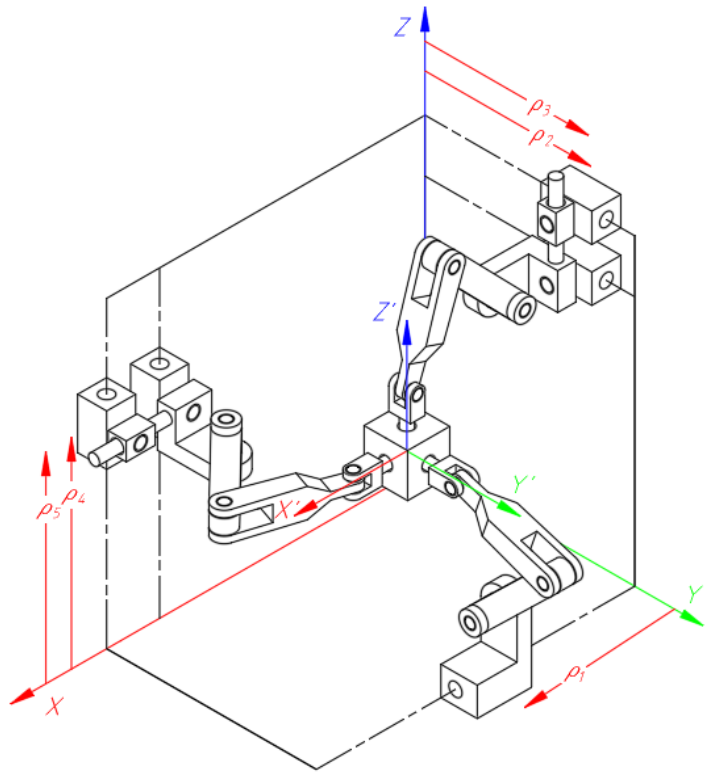

(a)

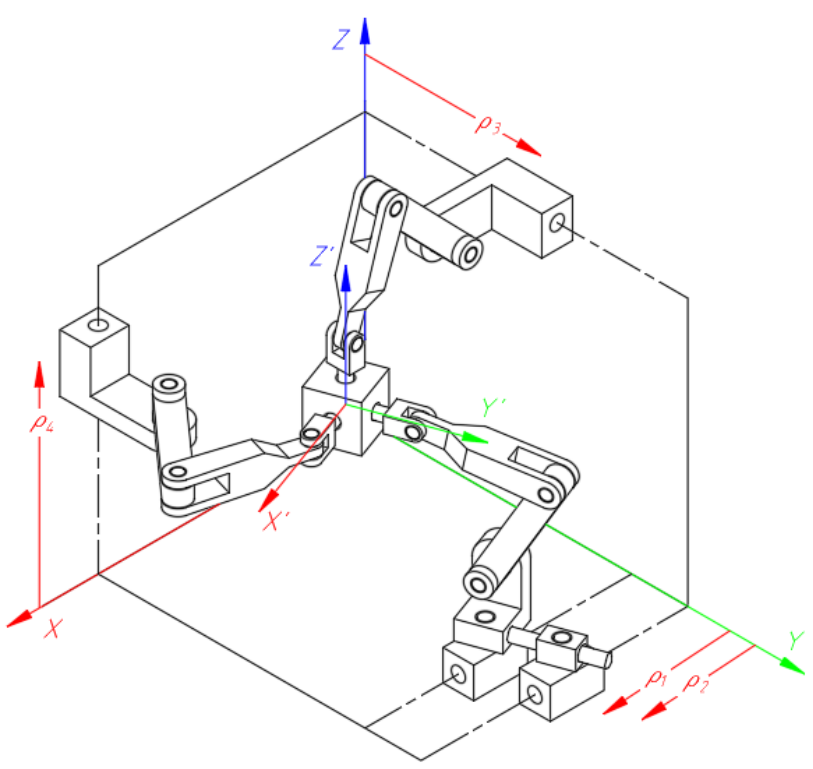

(b)

Fig. 7. Reduced-DOF alternative designs: (a) 5-DOF version, (b) 4-DOF version.

Finally, in this paper, we studied a design in which both the directions of the prismatic actuators and the axes of the platform revolute joints are orthogonal, respectively. Here, we propose two other geometries. In the first one, shown in Fig. 8(a), the angle between the directions of each pair of prismatic joints and the horizontal plane is $15^{\circ}$. Note that this angle cannot be $0^{\circ}$ or else the robot will always be in a singularity. However, the axes of the platform revolute joints are coplanar.

In the second geometry, shown in Fig. 8(b), the directions of the prismatic joints are vertical. In this design, the axes of the platform revolute joints are not coplanar, or else the robot would be always at a singularity. The angle between each pair of platform revolute joints is $110^{\circ}$.

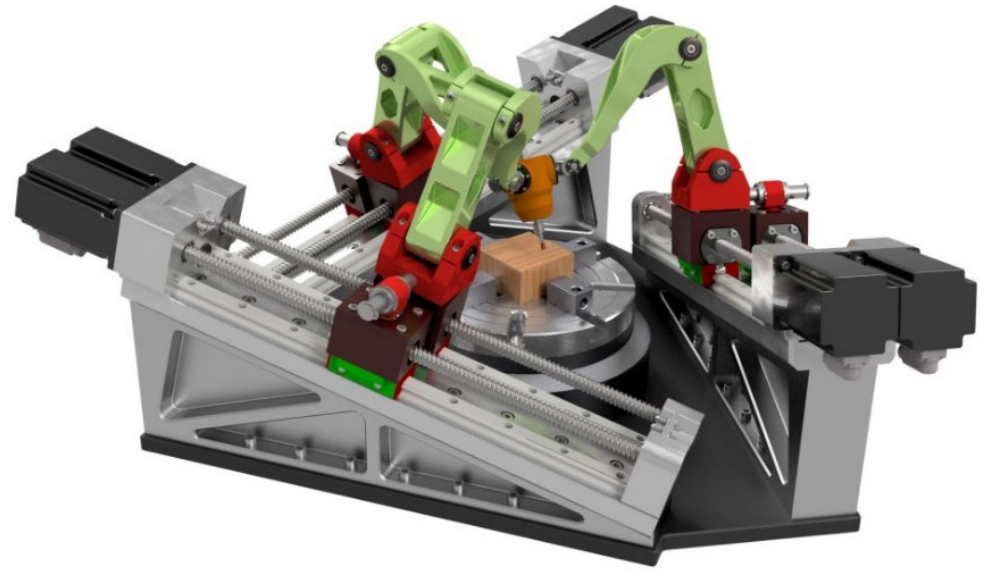

(a)

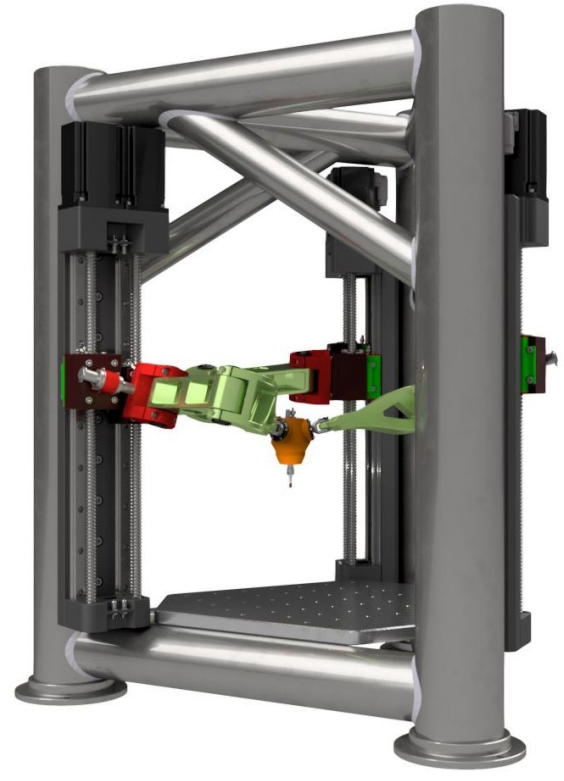

(b)

Fig. 8. Other designs with different orientations of the base prismatic joints and platform revolute joints. 
The equations for the inverse and direct kinematic problems for the proposed two new designs are similar to those shown in Section 2. However, the singularities of the new designs are substantially different. It is therefore crucial to perform an optimal design before selecting a specific design.

\section{CONCLUSIONS}

A novel three legged 6-DOF parallel robot was proposed. Its inverse and direct kinematics, and its singularity analyses are trivial and were presented in detail. Finally, 5-DOF and 4-DOF variants, as well as other 6-DOF designs with different angular dimensions were proposed.

\section{REFERENCES}

[1] X. Kong and C. M. Gosselin, "Kinematics and singularity analysis of a novel type of 3-CRR 3-DOF translational parallel manipulator," International Journal of Robotics Research, vol. 21, no. 9, pp. 791-798, 2002.

[2] C. M. Gosselin, X. Kong, S. Foucault and I. A. Bonev, "A fully decoupled 3-DOF translational parallel mechanism," 2004 Parallel Kinematic Machines International Conference, pp. 595-610, 2004.

[3] P. L. Richard, C. M. Gosselin and X. Kong, "Kinematic analysis and prototyping of a partially decoupled 4-DOF 3T1R parallel manipulator," in Proceedings of the 2006 ASME Design Engineering Technical Conferences \& Computers and Information in Engineering Conference, Philadelphia, USA, 2006.

[4] X. Kong and C. M. Gosselin, "Type synthesis of 5-DOF parallel manipulators based on screw theory," Journal of Robotic Systems, vol. 22, pp. 535-547, 2005.

[5] C. Gosselin, M. T. Masouleh, V. Duchaine, P. L. Richard and S. Foucault, "Parallel mechanisms of the Multipteron family: Kinematic architectures and benchmarking," in IEEE International Conference on Robotics and Automation, Roma, Italy, 2007.

[6] M. T. Masouleh, M. Husty and C. Gosselin, "Forward kinematic problem of 5-PRUR parallel mechanisms using study parameters," Advances in Robot Kinematics: Motion in Man and Machine, Springer, pp. 211-221, 2010.

[7] N. Seward and I. Bonev, "A new 6-DOF parallel robot with simple kinematic model," in International Conference on Robotics and Automation, Hong Kong, 2014.

[8] Yu, I. A. Bonev and P. Zsombor-Murray, "New XY-Theta positioning table with partially decoupled parallel kinematics," 2006 IEEE International Symposium on Industrial Electronics, Montreal, QC, Canada, July 9-13, 2006.

[9] Bonev, I.A., and Ryu, J., "A new approach to orientation workspace analysis of 6-DOF parallel manipulators," Mechanism and Machine Theory, Vol. 36, No. 1, pp. 15-28, 2001. 